\title{
LYMAN-ALPHA FOREST CONSTRAINTS ON THE MASS OF WARM DARK MATTER AND THE SHAPE OF THE LINEAR POWER SPECTRUM
}

\author{
Vijay K. Narayanan ${ }^{1}$, David N. Spergel ${ }^{1}$, Romeel Davé ${ }^{1,3}$, and Chung-Pei Ma ${ }^{2,4}$
}

\begin{abstract}
High resolution N-body simulations of cold dark matter (CDM) models predict that galaxies and clusters have cuspy halos with excessive substructure. Observations reveal smooth halos with central density cores. One possible resolution of this conflict is that the dark matter is warm (WDM); this will suppress the power spectrum on small scales. The Lyman-alpha forest is a powerful probe of the linear power spectrum on these scales. We use collisionless N-body simulations to follow the evolution of structure in WDM models, and analyze artificial Lyman-alpha forest spectra extracted from them. By requiring that there is enough small scale power in the linear power spectrum to reproduce the observed properties of the Lyman-alpha forest in quasar spectra, we derive a lower limit to the mass of the WDM particle of $750 \mathrm{eV}$. This limit is robust to reasonable uncertainties in our assumption about the temperature of the mean density gas $\left(T_{0}\right)$ at $z=3$. We argue that any model that suppresses the CDM linear theory power spectrum more severely than a $750 \mathrm{eV}$ WDM particle cannot produce the Lyman-alpha forest.
\end{abstract}

Subject headings: cosmology:theory, dark matter, methods:numerical, quasars: absorption lines

\section{Introduction}

Cold dark matter (CDM) models of structure formation have been remarkably successful in explaining a wide range of observations at both low and high redshifts. However, high resolution N-body simulations of the CDM model have revealed two potential conflicts with observations.

\footnotetext{
${ }^{1}$ Department of Astrophysical Sciences, Princeton University, Princeton, NJ 08544-1001; Email: vijay,dns,rad@astro.princeton.edu

${ }^{2}$ Department of Physics and Astronomy, University of Pennsylvania, Philadelphia PA 19104; Email: cpma@physics.upenn.edu

${ }^{3}$ Spitzer Fellow

${ }^{4}$ Alfred P. Sloan Fellow
} 
First, the inner mass density profiles of simulated halos are cuspy, as opposed to the shallow profiles inferred from the rotation curves of dwarfs and low surface brightness galaxies (Moore 1994; de Blok \& McGaugh 1997; Hernandez \& Gilmore 1998; but see van den Bosch et al. 1999), and the observations of soft cores in galaxy clusters by gravitational lensing (Tyson, Kochanski, \& DellÁntonio 1998). Second, group-sized halos in simulations contain a large number of low mass subhalos, greatly in excess of the observed number of satellite galaxies in the Local Group (Klypin et al. 1999; Moore et al. 1999). A variety of mechanisms have been proposed recently to reduce the clustering of mass on small scales, while simultaneously retaining the large scale successes of the CDM model. These mechanisms include breaking the scale invariance of the power spectrum of primordial density fluctuations (Kamionkowski \& Liddle 1999), postulating different collapse histories for dark matter and baryons (Navarro, Eke, \& Frenk 1996; Bullock, Kravtsov, \& Weinberg 2000; Binney, Gerhard, \& Silk 2000), and modifying the nature of dark matter. The last option includes dark matter models with a variety of properties - self-interacting (Sperge) \& Steinhardt 2000), warm (Sommer-Larsen \& Dolgov 1999) repulsive (Goodman 2000), fluid (Peebles 2000), and fuzzy (Hu, Barkana, \& Gruzinov 2000).

In the warm dark matter (WDM) model, the linear power spectrum is exponentially damped on scales smaller than the free-streaming length of the warm particle,

$R_{f}=0.2\left(\Omega_{\mathrm{W}} h^{2}\right)^{1 / 3}\left(m_{\mathrm{W}} / \mathrm{keV}\right)^{-4 / 3} \mathrm{Mpc}$, relative to the pure CDM model (Bardeen et al. 1986). Here, $\Omega_{\mathrm{W}}$ is the ratio of WDM density to the critical density, $m_{\mathrm{W}}$ is the mass of the WDM particle, and $h \equiv H_{0} / 100 \mathrm{~km} \mathrm{~s}^{-1} \mathrm{Mpc}^{-1}$ is the Hubble parameter. Non-linear gravitational evolution transfers power from large scales to small scales, so the clustering of the highly nonlinear mass distribution is insensitive to the shape of the linear power spectrum below the non-linear scale (Little, Weinberg, \& Park 1991; Bagla \& Padmanabhan 1997; White \& Croft 2000). Conversely, Ly- $\alpha$ absorbers seen in the spectra of high redshift $(z \sim 3)$ quasars arise from mass density fluctuations in the quasi-linear regime (Bi 1993; Hernquist et al. 1996; Hui \& Gnedin 1997), so their properties remain sensitive to the linear power spectrum. In this Letter, we set a lower limit on the mass of WDM particle by requiring that there be enough small scale power in the initial conditions to reproduce the observed properties of the Ly- $\alpha$ forest at $z=3$.

\section{Simulations}

We study the Ly- $\alpha$ forest in both CDM and WDM models with $\Omega_{m}=0.4, \Omega_{\Lambda}=0.6$, $h=0.65, \Omega_{b} h^{2}=0.02$, and $\sigma_{8 m}=0.95$, where $\Omega_{m}, \Omega_{\Lambda}$, and $\Omega_{b}$ are the contributions from total mass, vacuum energy, and baryons to the total energy density of the universe, and $\sigma_{8 m}$ is the rms density fluctuation in $8 h^{-1} \mathrm{Mpc}$ spheres, chosen here to reproduce the observed cluster abundance (White, Efstathiou, \& Frenk 1993; Eke, Cole, \& Frenk 1996). Table 1 lists the parameters of all models investigated. Our WDM models have $m_{\mathrm{W}}=200,500,750$, and $1000 \mathrm{eV}$ (corresponding to $R_{f}=0.95,0.28,0.16$, and $0.11 \mathrm{Mpc}$ ), spanning the range of WDM masses required to match the phase space density of dark matter cores in disk galaxies and dwarf spheroidal galaxies Hogan \& 
Dalcanton 2000 and references therein; Sellwood 2000) We also analyze a Broken Scale Invariance model (BSI, Kamionkowski \& Liddle 1999), using an analytic fit to its power spectrum from White \& Croft (2000), with a filtering scale $k_{0}=2 h \mathrm{Mpc}^{-1}$ required to fit the observed number density of low mass satellite galaxies in the Local Group.

We calculate the linear power spectrum of the mass density field for all the models using the full Boltzmann transport code of Ma \& Bertschinger (1995). We assume the usual three massless neutrino species with a present-day temperature $T_{0, \nu}=1.947 \mathrm{~K}$, and treat the WDM component as a fourth (massive) species with $T_{0, W}=T_{0, \nu}\left(\Omega_{W} h^{2}\right)^{1 / 3}\left(93 \mathrm{eV} / m_{W}\right)^{1 / 3}$. Fifty Legendre moments are used to follow the evolution of the WDM phase space. We compared our WDM power spectra with the fitting function of Bardeen et al. (1986) and find that their formula provides a reasonable approximation for $k<5 h \mathrm{Mpc}^{-1}$ [if a baryon-corrected shape parameter $\Gamma=\Omega_{m} h \exp \left(-\Omega_{b}\left(1+\Omega_{m}^{-1}\right)\right)$ is used], but it overestimates the power spectrum by up to an order of magnitude at higher $k$.

We employ a particle-mesh (PM) N-body code that is described in detail in Steed et al. (in preparation). This code computes forces using a staggered mesh (Melott 1986; Park 1990), and integrates the equations of motion using the leapfrog scheme described in Quinn et al. (1997). Our periodic simulation volume has $L=25 h^{-1} \mathrm{Mpc}$, with $N_{p}=256^{3}$ particles and an $N_{m}=512^{3}$ force mesh. We assign initial displacements and velocities to the particles using the Zeldovich approximation. In the WDM models, we also add a randomly oriented streaming velocity component that is drawn from a Fermi-Dirac distribution with an rms velocity, $v_{\text {rms }}=48.7\left(\frac{h}{0.65}\right)^{\frac{2}{3}}\left(\frac{m_{\mathrm{W}}}{100 \mathrm{eV}}\right)^{-\frac{4}{3}}\left(\frac{\Omega_{\mathrm{W}}}{0.4}\right)^{\frac{1}{3}}\left(\frac{1+z_{\mathrm{i}}}{50}\right) \mathrm{km} \mathrm{s}^{-1}$. We evolve from redshift $z=49 \rightarrow 3$ in 24 equal steps of the expansion scale factor.

Figure 1 shows the linear and non-linear power spectrum of the mass density field at $z=3$ in different models. The differences between different models are significantly smaller in the non-linear power spectra, compared to the differences in their linear theory power spectra, because non-linear gravitational evolution has regenerated power on small scales. Nevertheless, power transfer is not entirely successful in erasing the differences between the power spectra of different models. Thus, at $z=3$, the WDM200 model has almost an order of magnitude less power on small scales compared to the CDM model, while the models WDM500, WDM750, and BSIK02 are all deficient by $\sim 0.3$ dex. We also ran these models with a $50 h^{-1} \mathrm{Mpc}$ box using the same $N_{p}$ and $N_{m}$, and found similar results on overlapping length scales.

The model CDM200 has the same linear power spectrum as WDM200, but $v_{\text {rms }}=0$. This model gives virtually identical results to the WDM200 model, showing that the velocity dispersion of WDM particles has a negligible effect on the Ly- $\alpha$ forest at $z=3$. Hence, we do not show the results for this model in the figures. 

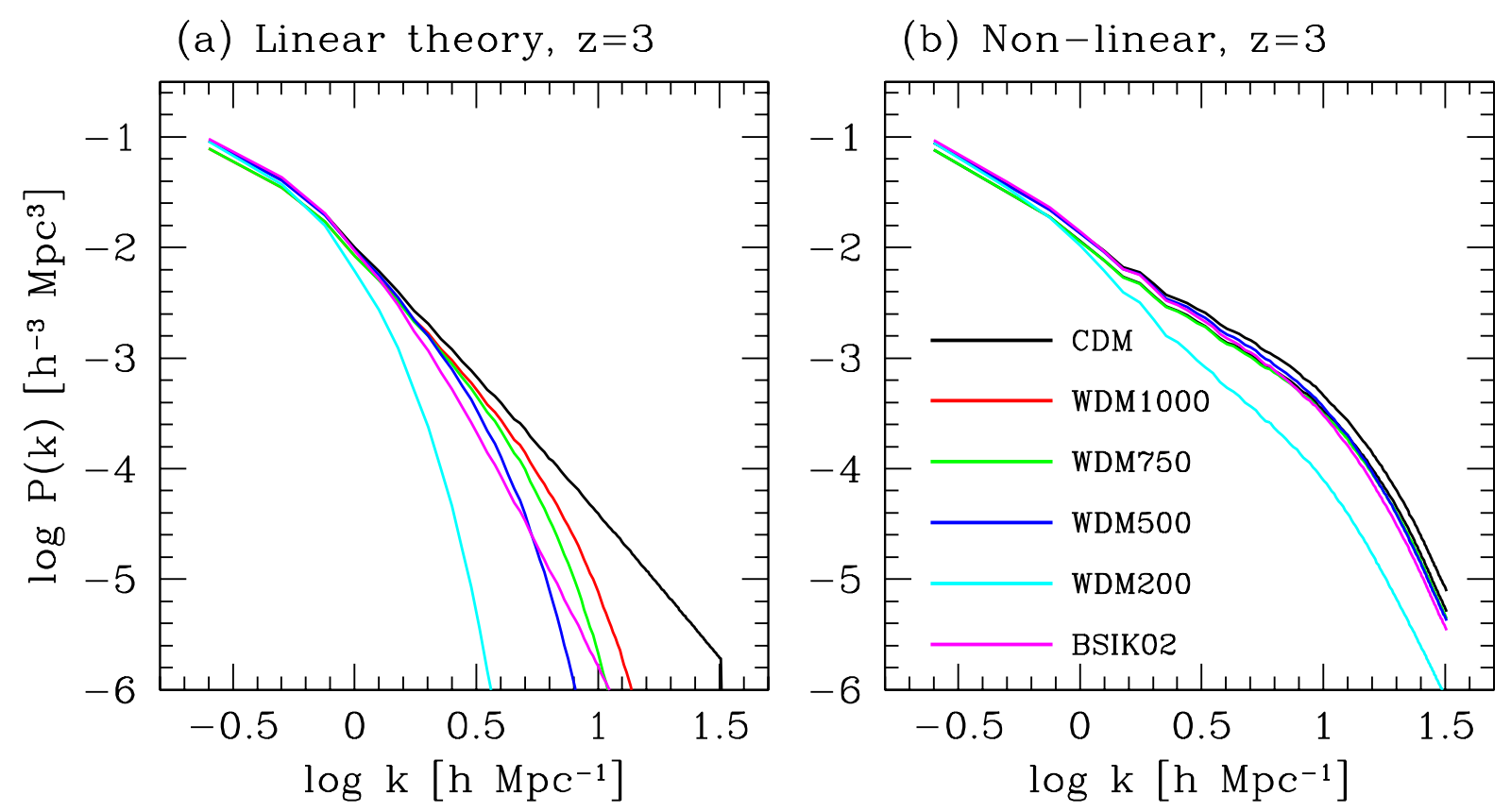

Fig. 1.- Power spectra of the mass density fields in different models. (a) Linear mass power spectra at $z=3$ from the Boltzmann code. (b) Non-linear power spectra at $z=3$ from simulation output, computed by cloud-in-cell binning onto a $256^{3}$ grid, taking its Fourier transform, and squaring the amplitudes of its Fourier components. Note that WDM750T025K has the same mass power spectrum as WDM750. 


\section{Properties of the Ly- $\alpha$ forest}

The Ly- $\alpha$ forest arises from a continuous, fluctuating, low-density $\left[-1 \lesssim \log \left(\rho_{\mathrm{g}} / \bar{\rho}_{\mathrm{g}}\right) \lesssim 1\right]$ gaseous medium whose temperature is determined by the balance between photoionization heating by the UV background and adiabatic cooling due to Hubble expansion (see e.g., Bi 1993; Cen et al. 1994; Zhang, Anninos, \& Norman 1995; Hernquist et al. 1996; Hui \& Gnedin 1997). Photoionization equilibrium leads to a power-law relation between temperature and gas density, of the form $T=T_{0}\left(\rho_{g} / \bar{\rho}_{g}\right)^{\alpha}$, with $4000 \mathrm{~K} \leq T_{0} \leq 10000 \mathrm{~K}$, and $0.3 \leq \alpha \leq 0.6$ Hui \& Gnedin 1997). The optical depth to Ly- $\alpha$ photons $\left(\tau_{\mathrm{Ly} \alpha}\right)$ is proportional to H I gas density, which in turn is proportional to $T^{-0.7}$ for gas in photoionization equilibrium near $10^{4} \mathrm{~K}$. Pressure gradients in this low-density, fairly cool $\left(T \sim 10^{4} \mathrm{~K}\right)$ gas are small, so gas traces dark matter (Croft et al. 1998). Hence, the gas density-temperature relation can be translated into a relation between mass density $\left(\rho_{m}\right)$ and $\tau_{\text {Ly } \alpha}$ (the "Fluctuating Gunn-Peterson Approximation", Rauch et al. 1997; Croft et al. 1998; Weinberg, Katz, \& Hernquist 1998). Thus, the transmitted flux of Ly- $\alpha$ photons $\left[\mathrm{F}=\exp \left(-\tau_{\mathrm{Ly} \alpha}\right)\right]$ in a quasar spectrum is a map of the mass density fluctuations along the line of sight.

We extract artificial Ly- $\alpha$ absorption spectra along 400 random lines of sight through each simulation, using the TIPSY package (Katz \& Quinn 1995; see http://wwwhpcc.astro.washington.edu/tools/TIPSY]. TIPSY calculates the local mass density $\left(\rho_{m}\right)$ at the position of each dark matter particle using a cubic spline smoothing kernel (Hernquist \& Katz 1989) enclosing 32 neighbors, and assigns it a temperature $T=T_{0}\left(\rho_{m} / \bar{\rho}_{m}\right)^{0.6}$. Spectra are then computed from this particle distribution using the algorithm described by Hernquist et al. (1996). We sample the spectra with resolution $\Delta v=6.4 \mathrm{~km} \mathrm{~s}^{-1}$, roughly corresponding to that of the Keck HIRES spectra to which we will compare our model predictions. We "fit a continuum" to each spectral segment, $3200 \mathrm{~km} \mathrm{~s}^{-1}$ in length, by rescaling all flux values so that the highest pixel has transmission of unity (Davé et al. 1999). Fitting a continuum makes low-power models appear more like high power models, so it is an important step for conservatively discriminating against models with suppressed power.

The optical depth to Ly- $\alpha$ photons depends on a number of uncertain parameters including $\Omega_{b}, h, T_{0}$, and the intensity of the ionizing background. However, the cumulative effect of all these parameters is to scale the normalization factor $(A)$ relating $\tau_{\text {Ly } \alpha}$ to $\rho_{m} / \bar{\rho}_{m}$, as long as collisional ionization is unimportant (Weinberg et al. 1998). We fix $A$ in each model so that the mean transmitted flux at $z=3, \overline{\mathrm{F}}=\left\langle\exp \left(-\tau_{\mathrm{Ly} \alpha}\right)\right\rangle$, is 0.684 , as measured by McDonald et al. (1999) from Keck HIRES spectra of eight quasars.

Figure 2 shows artificial Ly- $\alpha$ absorption spectra at $z=3$ extracted along four random lines of sight, in three different models: CDM, WDM500, and WDM750. The gas responsible for Ly- $\alpha$ absorption is in larger and more diffuse structures in WDM models compared to the CDM model. Hence, the WDM spectra are smoother and have less small scale power as compared to the CDM spectra. The fluctuations in the absorbing gas are also weaker in the WDM models, resulting in 
absorption lines that are broader and shallower than corresponding lines in the CDM artificial spectra.

Figure 3 shows the one dimensional power spectrum of the transmitted flux, $P_{F}(k)$, computed using 400 artificial spectra for each model. The solid points show $P_{F}(k)$ measured by McDonald et al. (1999). The shape and amplitude of $P_{F}(k)$ of the CDM model matches the observations very well, in the entire range $-2.5 \lesssim \log \left[k\left(\mathrm{~km}^{-1} s\right)\right] \lesssim-1$. However, the WDM models predict a wrong shape of $P_{F}(k)$. Thus, although the $P_{F}(k)$ of WDM models match the observations on large

scales, $-2.5 \lesssim \log \left[k\left(\mathrm{~km}^{-1} s\right)\right] \lesssim-2$, they have too little power on smaller scales. This confirms the visual impression from the spectra in Fig. 2, and is also evident in the flux decrement correlation function (not shown). A higher value of $T_{0}$ further suppresses $P_{F}(k)$ on smaller scales, worsening the discrepancy with observations. Note that lowering $\Omega_{m}$ to make up the power suppressed by WDM is not feasible; a lower $\Omega_{m}$ raises the power spectrum on all scales at $z=3$, and thus would be inconsistent with the observed $P_{F}(k)$ on large scales.

Figure 4 shows the probability distribution function (PDF) of the transmitted flux (Jenkins \& Ostriker 1991; Miralda-Escude et al. 1996; Rauch et al. 1997), computed using 400 artificial spectra for each model. The solid points show the flux PDF measured by McDonald et al. (1999). The flux PDF of the CDM model matches observations remarkably well over the entire range of flux values. As the mass of WDM decreases, there is less small scale power in the mass distribution, the mass fluctuations are weaker, and the gas responsible for Ly- $\alpha$ absorption is in more diffuse structures. Hence there are fewer pixels where the transmission approaches unity, and more pixels at intermediate fluxes. In the flux range $0.6<\mathrm{F}<0.8$, the flux PDF for all WDM models with $m_{\mathrm{W}} \leq 750 \mathrm{eV}$ differs from observations by $\gtrsim 3 \sigma$. This mismatch further confirms that the mass fluctuation amplitude of these WDM models is too low at small scales when the fluctuations are normalized to match $P_{F}(k)$ on large scales. Even the model WDM750T025K, with a higher temperature for the mean-density gas $\left(T_{0}=25,000 \mathrm{~K}\right)$, does not match the observations as well as the CDM model. On the other hand, while WDM1000 does not match the observations very well, a higher value of $T_{0}$ can bring its flux PDF to better agreement with observations. Since recent determinations of $T_{0}$ prefer values around 20,000 K (e.g., Schaye et al. 1999), we conclude that the observed flux PDF cannot be reproduced by WDM models with $m_{\mathrm{W}} \lesssim 750 \mathrm{eV}$. The BSIK02 model is also inconsistent with observations, being similar to WDM500.

\section{Conclusions}

For a given cosmology, the properties of the Ly- $\alpha$ forest depend on three factors: (a) the linear theory power spectrum, taken from a Boltzmann code; (b) power transfer from large to small scales during non-linear gravitational evolution, followed using N-body simulations; and (c) the equation of state of the intergalactic medium, governed by photoionization equilibrium. Here, we have used the observed Ly- $\alpha$ forest at $z=3$ to constrain the shape of the linear power spectrum, and consequently $m_{\mathrm{W}}$. Our principal conclusions are as follows: 


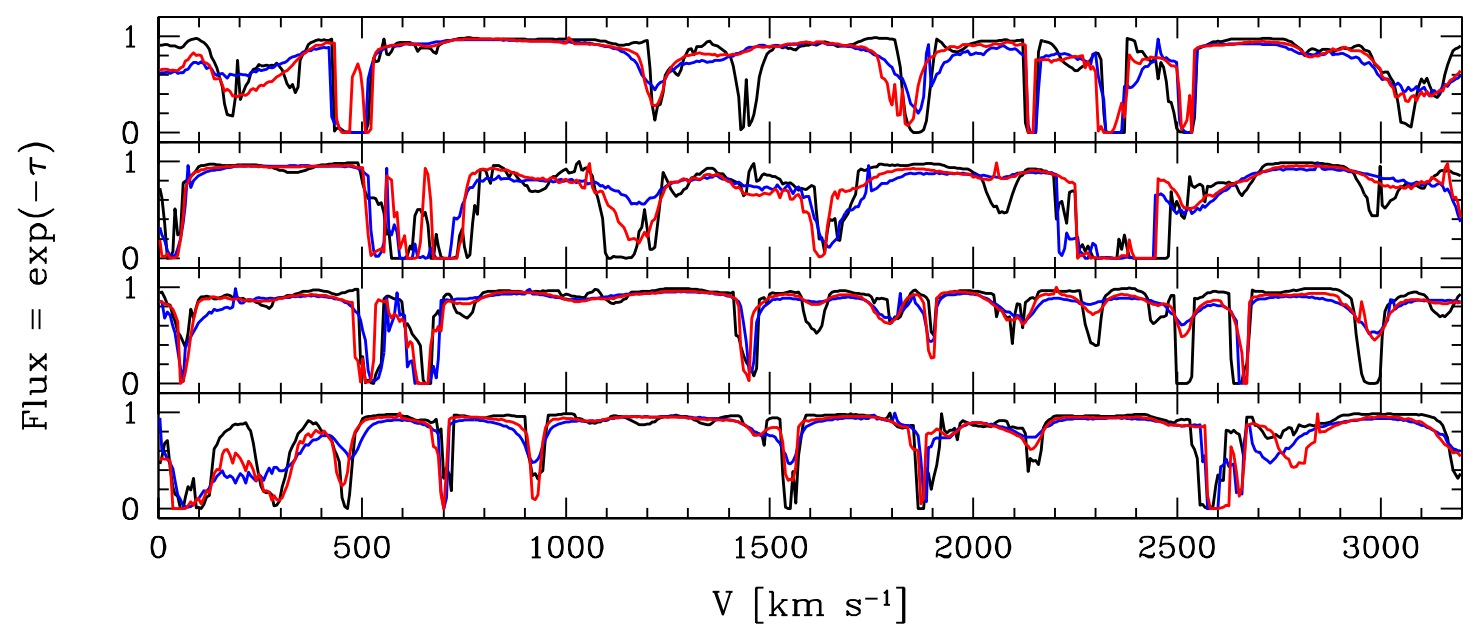

Fig. 2. - Examples of artificial spectra at $z=3$ along four random lines of sight in the simulations. All simulations have identical phases for the Fourier components of the initial density fields. In each panel, black line shows results for CDM, blue line shows WDM500, and red line shows WDM750. 


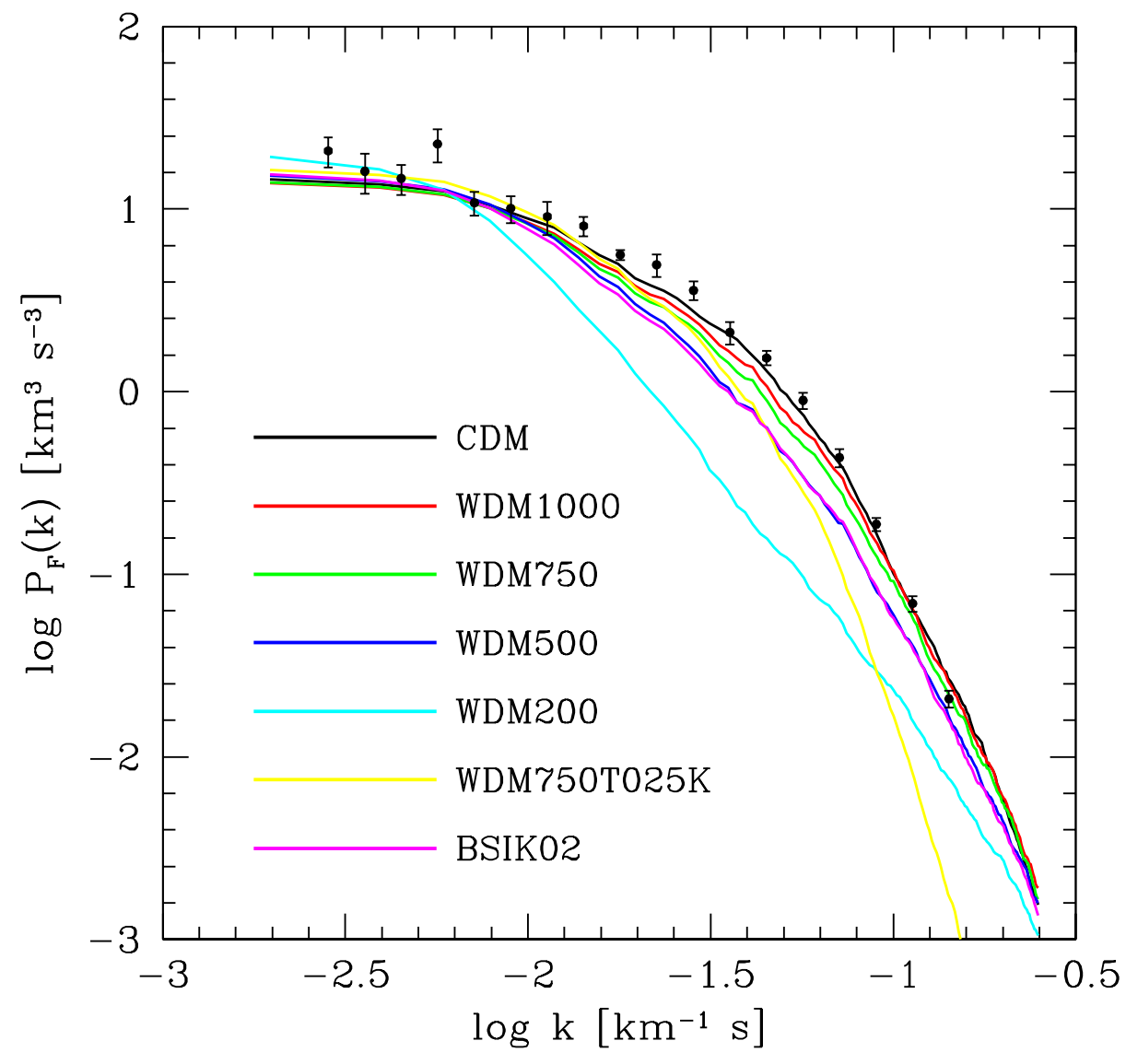

Fig. 3.- Power spectrum of the transmitted flux at $z=3$ from 400 artificial spectra for each model. Solid points with error bars show the flux power spectrum at $z \approx 3$ measured by McDonald et al. (1999) from eight Keck HIRES quasar spectra. 


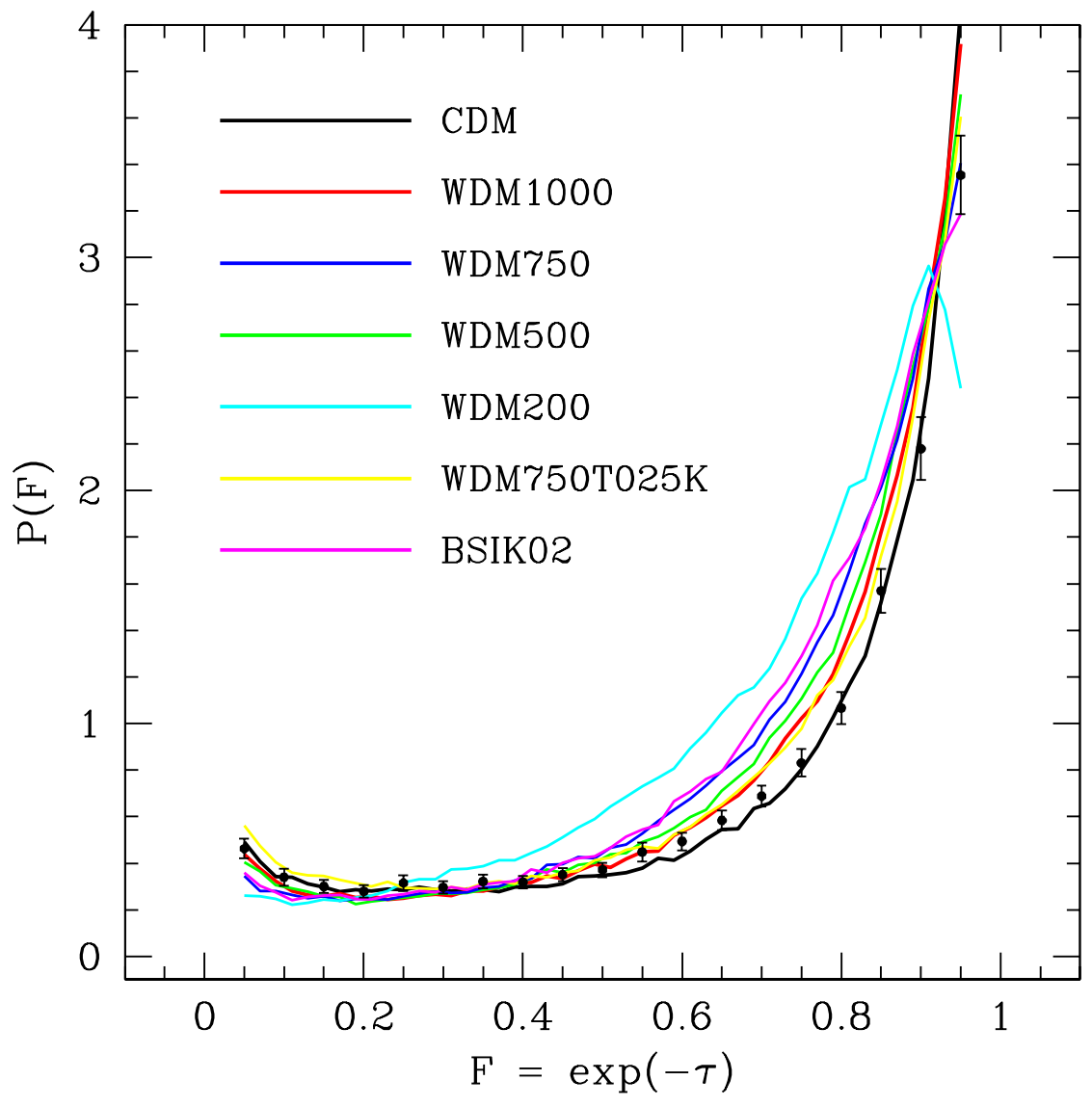

Fig. 4.- Probability distribution functions (PDF) of the transmitted flux at $z=3$, computed using 400 artificial spectra for each model. Solid points with error bars show the flux PDF at $z=3$ measured by McDonald et al. (1999) using Keck HIRES spectra of eight quasars. 
(1) $m_{\mathrm{W}} \gtrsim 750 \mathrm{eV}$ is required to match $P_{F}(k)$ and flux PDF of artificial spectra extracted from simulations of WDM models with Keck HIRES observations of McDonald et al. (1999), for any reasonable $T_{0}$. This limit is similar to that obtained from the maximum observed phase space density of dwarf spheroidal galaxies (Dalcanton \& Hogan 2000). High resolution N-body simulations of WDM models with $m_{\mathrm{W}} \sim 1 \mathrm{keV}$ predict the observed circular velocity function of satellite galaxies in the Local Group, but produce cuspy halos (Colin, Avila-Reese, \& Valenzuela 2000).

(2) Ly- $\alpha$ forest properties depend on the shape of the linear power spectrum, and are insensitive to streaming velocities of WDM particles. Even for a $200 \mathrm{eV}$ WDM particle, $v_{\mathrm{rms}}=1.5 \mathrm{~km} \mathrm{~s}^{-1}$ at $z=3$, an order of magnitude smaller than the thermal velocity of $\mathrm{H}$ I gas at $T \sim 10^{4} \mathrm{~K}$. Hence, any mechanism that suppresses the CDM linear power spectrum more severely than a $750 \mathrm{eV}$ WDM particle will be inconsistent with Ly- $\alpha$ forest observations.

(3) The BSI model proposed by Kamionkowski \& Liddle (1999), with $k_{0}=2 h \mathrm{Mpc}^{-1}$ chosen to match the abundance of dwarf galaxies, does not reproduce the observed properties of the Ly- $\alpha$ forest at $z=3$. We note that this is the extreme BSI model considered by White \& Croft (2000), hence our conclusion is not in conflict with theirs.

We thank David Weinberg and Michael Strauss for helpful comments on the manuscript. DNS is partially supported by the MAP/MIDEX program, and the NASA ATP grant NAG5-7154. CPM is supported by a Cottrell Scholars Award from the Research Corporation, and NSF grant AST 9973461.

\section{REFERENCES}

Bagla, J. S., \& Padmanabhan, T. 1997, MNRAS, 286, 1023

Bardeen, J., Bond, J. R., Kaiser, N., \& Szalay, A. 1986, ApJ, 304, 15

Bi, H., 1993, ApJ, 405, 479

Bi, H., \& Davidsen, A. F., 1997, ApJ, 479, 523

Binney, J. J., Gerhard, O., \& Silk, J., 2000, MNRAS, submitted (astro-ph/0003199)

Bullock, J. S., Kravtsov, A. V., \& Weinberg, D. H, 2000, ApJ, submitted (astro-ph/0002214)

Colin, P., Avila-Reese, V., \& Valenzuela, O., 2000, ApJ, submitted astro-ph/0004115)

Cen, R., Miralda-Escude, J., Ostriker, J. P., \& Rauch, M., 1994, ApJ, 437, L9

Croft, R. A. C., Weinberg, D. H., Katz, N., \& Hernquist, L. 1998, ApJ, 495, 44

Dalanton, J. J,. \& Hogan, C. J., 2000, preprint (astro-ph/0004381) 
Davé, R., Hernquist, L., Katz, N., \& Weinberg, D. H., 1999, ApJ, 511, 521

de Blok, W. J. G., \& McGaugh, S. S., 1997, MNRAS, 290, 533

Eke, V. R., Cole, S., Frenk, C. S. 1996, MNRAS, 282, 263

Flores, R., \& Primack, J. R., 1994, ApJ, 427, L1

Goodman, J., 2000, preprint (astro-ph/0003018)

Hernandez, X., \& Gilmore, G., 1998, MNRAS, 294, 595

Hernquist, L., \& Katz, N. 1989, ApJS, 70, 419

Hernquist L., Katz, N., Weinberg, D. H., \& Miralda-Escudé, J. 1996, ApJ, 457, L5

Hogan, C. J., \& Dalanton, J. J., 2000, preprint (astro-ph/0002330)

Hu, W., Barkana, R., \& Gruzinov, A., 2000, Physics Review Letters, submitted (astro-ph/0004151)

Hui, L., \& Gnedin, N. Y., 1997, MNRAS, 292, 27

Jenkins, E. B., \& Ostriker, J. P., 1991, ApJ, 376, 33

Kamionkowski, M., \& Liddle, A. R., 1999, preprint (astro-ph/9911103)

Katz, N., \& Quinn, T., 1995, TIPSY manual

Klypin, A., Kravtsov, A. V., Valenzuela, O., \& Prada, F., 1999, ApJ, 522, 82

Little, B., Weinberg, D. H, \& Park, C. 1991, MNRAS, 253, 295

Ma, C. P., \& Bertschinger, E. B., 1995, ApJ, 455, 7

McDonald, P., Miralda-Escudé, J., Rauch, M., Sargent, W. L. W., Barlow, T. A., Cen, R., \& Ostriker, J. P., ApJ, submitted (astro-ph/9911196)

Mirald-Escude, J., Cen, R., Ostriker, J. P., \& Rauch, M., 1996, ApJ, 471, 582

Melott, A. L., 1986, Physics Review Letters, 56, 1992

Moore, B., 1994, Nature, 370, 629

Moore, B., Ghigna, S., Governato, F., Lake, G., Quinn, T., Stadel, J., \& Tozzi, P., 1999, ApJ, 524, L19

Moore, B., Gelato, S., Jenkins, A., Pearce, F., \& Quilis, V., 2000, MNRAS, submitted (astro-ph/0002308)

Navarro, J. F., Eke, V. R., \& Frenk, C. S., 1996, MNRAS, 283, L72 
Park, C., Ph.D Thesis, Princeton University.

Peebles, P. J. E., 2000, preprint (astro-ph/0002495)

Quinn, T., Katz, N., Stadel, J., \& Lake, G., 1997, preprint (astro-ph/9710043)

Rauch, M., et al., 1997, ApJ, 489, 7

Schaye, J., Theuns, T., Rauch, M., Efstathiou, G., \& Sargent, W. L. W., 1999, MNRAS, submitted (astro-ph/9912432

Sellwood, J. A., 2000, ApJ, submitted (astro-ph/0004352)

Sommer-Larsen, J., \& Dolgov, A., 1999, ApJ, submitted (astro-ph/9912166

Spergel, D. N., \& Steinhardt, P. J., 2000, Physics Review Letters, in press

Tyson, J. A., Kochanski, G. P., \& DellÁntonio, I. P., 1999, ApJ, 498, L107

van den Bosch, F., Robertson, B. E., Dalcanton, J. J., \& de Blok, W. J. G., 1999, AJ, submitted (astro-ph/9911372

Weinberg, D. H., Katz, N., \& Hernquist, L. 1998, in ASP Conference Series 148, Origins, eds. C. E. Woodward, J. M. Shull, \& H. Thronson, (ASP: San Francisco), 21, astro-ph/9708213

Weinberg, D. H., et al. 1998, in Proceedings of the MPA/ESO Conference "Evolution of Large Scale Structure: From Recombination to Garching"

White, M., \& Croft, R. A. C., 2000, ApJ, submitted (astro-ph/0001247)

White, S. D. M., Efstathiou, G. P., \& Frenk, C. S. 1993, MNRAS, 262, 1023

Zhang, Y., Anninos, P., \& Norman, M. L., 1995, ApJ, 453, L57 
Table 1: Model parameters.

\begin{tabular}{llccc}
\hline \hline Model & Power spectrum & $\begin{array}{c}m_{\mathrm{W}} \\
(\mathrm{eV})\end{array}$ & $\begin{array}{c}v_{\mathrm{rms}}\left(z_{i}=49\right) \\
\left(\mathrm{km} \mathrm{s}^{-1}\right)\end{array}$ & $\begin{array}{c}T_{0}(z=3) \\
(\mathrm{K})\end{array}$ \\
\hline CDM & CDM & - & 0 & 6000 \\
WDM1000 & WDM & 1000 & 2.3 & 6000 \\
WDM750 & WDM & 750 & 3.3 & 6000 \\
WDM500 & WDM & 500 & 5.7 & 6000 \\
WDM200 & WDM & 200 & 19.2 & 6000 \\
CDM200 & WDM $^{*}$ & 200 & 0 & 6000 \\
WDM750T025K & WDM $_{\text {BSIK02 }}$ & 750 & 3.3 & 25000 \\
& $\mathrm{CDM}, B S I^{\dagger}$ & - & 0 & 6000 \\
\hline
\end{tabular}

*This model has the same linear power spectrum as WDM200.

${ }^{\dagger}$ We use an analytic fit to the power spectrum of the Broken Scale Invariance model with $k_{0}=2 h \mathrm{Mpc}^{-1}$, given by White \& Croft 2000. 